\title{
Dynamics Analysis of planetary Gear Train with Two Degrees of Freedom
}

\author{
Lin Xu, Kai Liu, Yahui Cui and Peiyuan Meng \\ School of Mechanical and Precision Instrument Engineering, Xi'an University of Technology, Xi'an \\ 710048, China \\ vsxulin@gmail.com
}

Keywords: Planetary gear train; Equivalent moment of inertia; Dynamic response; Torque ratio; Power ratio

\begin{abstract}
Dynamic analysis method is used in this paper to analyze the dynamic response of planetary gear train with two degrees of freedom under the conditions of the start-up and load step change during operation. The results show that the angular velocity relationship between the components under steady-state and non steady-state conditions follows the rules of characteristic parameters, $\mathrm{k}$. The torque follows the rules related to the $\mathrm{k}$ only under the steady-state, but not consistent under the non-steady state conditions. The results also show that the power distribution ratio of differential gear train is not a fixed parameters value, but relevant to the operation status. The research further improved the basic gear train's translation theory.
\end{abstract}

\section{Introduction}

Gear transmission has been extensively used in machines and equipments, because of its high efficiency, compact structure, reliable operation, long service life and the advantages of flexible modes of transmission. Gear transmission is divided into ordinary gear train and planetary gear train(PGT). Compared with ordinary gear train, PGT has many advantages such as smaller volume, larger carrying capacity, larger transmission ratio.

After study of published papers, PGT has been investigated in two different aspects by researchers. The first aspect is the static characteristic that has been comprehensively studied and verified by experiments [1-3]. The second aspect is the dynamic characteristic. LUO [4] proposed a dynamic model based on bound graph theory and carried out a free torsional vibration analysis as an illustration of the model's application in modal analysis. QIN [5] used vibration superposition method to analyze the critical components' vibration displacements of a wind turbine. LIN [6,7] analyzed PGT's free vibration and the parameter instability caused by mesh stiffness changing. BAHK et al. [8,9] established a tooth profile modification model to study on nonlinear dynamic of PGT. The amount of tooth profile modification was changed to analyze its effect on vibration. SUN et al. [10] analyzed the nonlinear vibration of a 2K-H PGT excited by a static transmission error in addition to an external torque. In LIU's paper [11], he specialized in the influence of parameters which included time-varying mesh stiffness, gear backlashes and synthetic mesh errors to vibration characteristics. ZHU et al. [12] studied the vibration response caused by time-varying mesh stiffness, synthetic mesh errors and sliding friction. GUO et al. [13, 14] established a PDT model including gravity, fluctuating mesh stiffness, bearing clearance, and nonlinear tooth contact to obtain the vibration response of the system. These papers focused on the vibration response of the PGT and the sources of vibration.

MANGIALARDI [15] used static behavior instead of dynamic behavior in each operating condition when he was analyzing infinitely variable transmission's torque ratio and efficiency performance. But in practical applications, dynamic behavior is different from static behavior, it is improper to replace dynamic process with static solution. This paper uses dynamic analysis method to examine PGT's different operation of different conditions. 


\section{Dynamic Analysis of PGT}

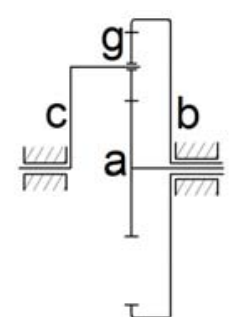

(a) Schematic of PGT

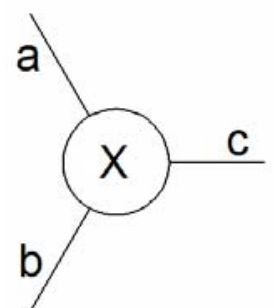

(b) simplified diagram of PGT

Fig.1 Schematic and simplified diagram of PGT

Fig. 1(a) shows the schematic of PGT, while (b) is the simplified diagram. PGT has three basic components, which are represented by letters of $\mathrm{a}, \mathrm{b}$ and $\mathrm{c}$, and performed as input and output function. Planetary gear is represented by g, and PGT is represented by X.

Kinetic energy of PGT. PGT has two degrees of freedom, according to Lagrange method, hence, we need two generalized coordinates for description. We select angular displacements of components, $a$ and $b$, as the generalized coordinates, i.e. $\mathrm{q}_{1}=\varphi_{\mathrm{a}}, \mathrm{q}_{2}=\varphi_{\mathrm{b}}$. Corresponding to which generalized angular velocities are

$$
\left\{\begin{array}{l}
\dot{q}_{1}=w_{a} \\
\dot{q}_{2}=w_{b}
\end{array}\right.
$$

Define the characteristic parameter $\mathrm{K}$ as transmission ratio of PGT:

$$
i_{a b}^{c}=\frac{w_{a}-w_{c}}{w_{b}-w_{c}}=K
$$

And the angular velocity of component $\mathrm{c}$ is:

$$
w_{c}=\frac{1}{1-K} \dot{q}_{1}-\frac{K}{1-K} \dot{q}_{2}
$$

Components $\mathrm{a}, \mathrm{b} \& \mathrm{c}$ rotate around their axis respectively, thus their kinetic energy is generated by rotation only. Planetary gear g's velocity contains two aspects, i.e. rotation velocity and revolution velocity, thus the kinetic energy also has two parts. The revolution velocity equals to component $\mathrm{c}$, the rotation velocity can be calculated as:

$$
w_{g}^{c}=w_{g}-w_{c}=\frac{2 K}{1-K^{2}}\left(\dot{q}_{2}-\dot{q}_{1}\right)
$$

Thus PGT's kinetic energy becomes:

$$
\begin{aligned}
E_{K} & =\frac{1}{2} J_{a} \cdot w_{a}^{2}+\frac{1}{2} J_{b} \cdot w_{b}^{2}+\frac{1}{2} J_{c} \cdot w_{c}^{2}+\frac{1}{2} J_{g} \cdot w_{g}^{c 2}+\frac{1}{2} \cdot n \cdot m_{g}\left(R_{a}+R_{g}\right)^{2} w_{c}^{2} \\
& =\frac{1}{2} J_{11} \cdot \dot{q}_{1}^{2}+J_{12} \cdot \dot{q}_{1} \cdot \dot{q}_{2}+\frac{1}{2} J_{22} \cdot \dot{q}_{2}^{2}
\end{aligned}
$$

Equivalent moments of inertia $\mathrm{J}_{11}, \mathrm{~J}_{12}, \mathrm{~J}_{22}$ are calculated by:

$$
\left\{\begin{array}{l}
J_{11}=J_{a}+\frac{1}{(1-k)^{2}} \cdot J_{c}+4 \frac{k^{2}}{\left(k^{2}-1\right)^{2}} \cdot J_{g}+J_{o} \\
J_{12}=\frac{-k}{(1-k)^{2}} \cdot J_{c}-4 \frac{k^{2}}{\left(k^{2}-1\right)^{2}} \cdot J_{g}-k \cdot J_{o} \\
J_{22}=J_{b}+\frac{k^{2}}{(1-k)^{2}} \cdot J_{c}+4 \frac{k^{2}}{\left(k^{2}-1\right)^{2}} \cdot J_{g}+k^{2} \cdot J_{o}
\end{array}\right.
$$

$\mathrm{J}_{\mathrm{a}}, \mathrm{J}_{\mathrm{b}}, \mathrm{J}_{\mathrm{c}}$, and $\mathrm{J}_{\mathrm{g}}$ represent components $\mathrm{a}, \mathrm{b}$, c and planetary gear g's moment of inertia respectively. $\mathrm{J}_{0}=\mathrm{n} \cdot \mathrm{m}_{\mathrm{g}} \cdot\left(\mathrm{R}_{\mathrm{a}}{ }^{2}\right) / 4$ has no relation with speed but is determined by structure parameters, so it is represented by $\mathrm{J}_{0}$.

Generalized force of PGT. The power of PGT can be expressed as 


$$
P=T_{a} \cdot \omega_{a}+T_{b} \cdot \omega_{b}+T_{c} \cdot \omega_{c}
$$

So the generalized force are:

$$
\left\{\begin{array}{l}
F_{1}=T_{a}+\frac{R_{a}}{R_{a}+R_{b}} T_{c} \\
F_{2}=T_{b}+\frac{R_{b}}{R_{a}+R_{b}} T_{c}
\end{array}\right.
$$

$\mathrm{T}_{\mathrm{a}}, \mathrm{T}_{\mathrm{b}}, \mathrm{T}_{\mathrm{c}}$ are external torques acting on components $\mathrm{a}, \mathrm{b}$, and $\mathrm{c} ; \mathrm{R}_{\mathrm{a}}, \mathrm{R}_{\mathrm{b}}, \mathrm{R}_{\mathrm{c}}$ are radius of components $\mathrm{a}, \mathrm{b}$, and $\mathrm{c}$.

Motion differential equations of PGT. The Lagrange equation of the two degrees of freedom system [16] is

$$
\frac{d}{d t}\left(\frac{\partial E_{k}}{\partial \dot{q}_{i}}\right)-\frac{\partial E_{k}}{\partial q_{i}}+\frac{\partial E_{p}}{\partial q_{i}}=F_{i}, \quad(i=1,2)
$$

In the formula: $E_{k}$ denotes the kinetic energy of the system; $E_{p}$ denotes the potential of the system; $\mathrm{q}_{\mathrm{i}}$ are generalized coordinates. Solve Eq. (9) by Eqs. (2)-(8), then we can sort out the motion differential equations of PGT:

$$
\left\{\begin{array}{l}
F_{1}=J_{1} \cdot \ddot{q}_{1}+J_{12} \cdot \ddot{q}_{2} \\
F_{2}=J_{12} \cdot \ddot{q}_{1}+J_{2} \cdot \ddot{q}_{2}
\end{array}\right.
$$

\section{Calculation and Analysis of Results}

A PGT with $\mathrm{K}=0.73$ is selected as the object to analyze the performance in the conditions of boot and load mutation during stable operation. We get the following results.

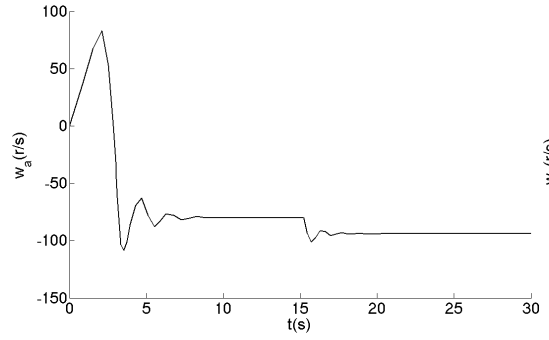

(a) angular velocity of a

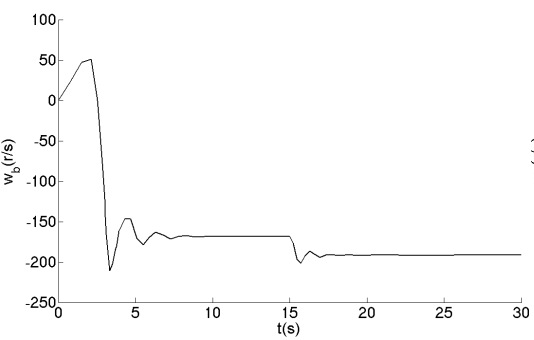

(b) angular velocity of $b$

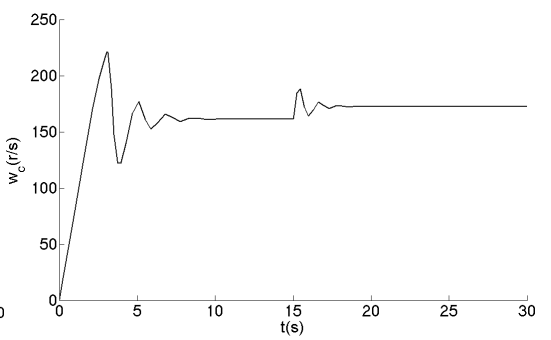

(c) angular velocity of c

Fig. 2 angular velocity of PGT's components

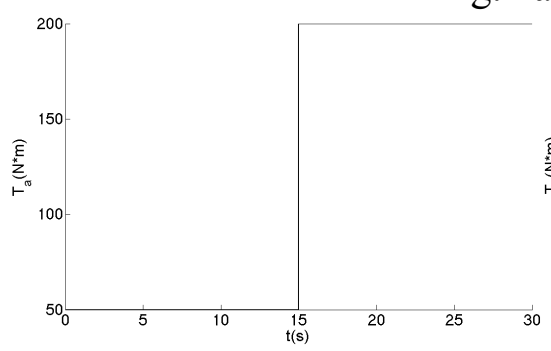

(a) torque of a

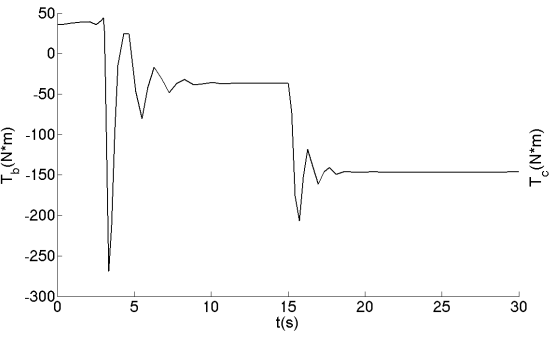

(b) torque of $b$

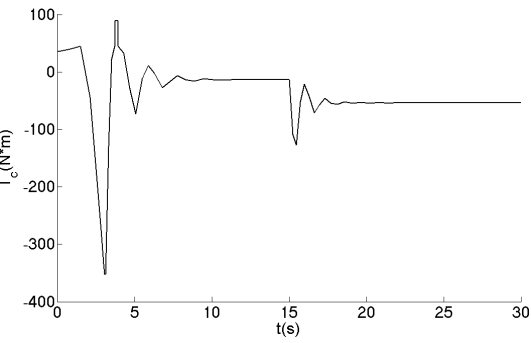

(c) torque of c

Fig.3 torque of PGT's components

Tab. 1 angular velocities of 4 periods

\begin{tabular}{ccccc}
\hline & $\mathrm{t}_{1}$ & $\mathrm{t}_{2}$ & $\mathrm{t}_{3}$ & $\mathrm{t}_{4}$ \\
\hline$\omega_{\mathrm{a}}(\mathrm{r} / \mathrm{s})$ & -56.635 & -80 & -92.55 & -96 \\
$\omega_{\mathrm{b}}(\mathrm{r} / \mathrm{s})$ & -158.8 & -168.29 & -195.1 & -191.5 \\
$\omega_{\mathrm{c}}(\mathrm{r} / \mathrm{s})$ & 221 & 161.535 & 188 & 162.61 \\
$\mathrm{~K}_{\mathrm{i}}$ & 0.73 & 0.73 & 0.73 & 0.73 \\
\hline
\end{tabular}

Tab. 1 extracts the angular velocities at random moments of each period $(t 1, t 2, t 3, t 4) . K_{i}$ denotes the corresponding transmission ratio. The calculation results show that every $\mathrm{K}_{\mathrm{i}}$ equals to characteristic parameter $\mathrm{K}$. It proves that the ratio of angular velocities in dynamic process consistent with the ratio of static analysis result. 
Tab. 2 extracts the torques at random moments of each period. According to static analysis, we have the relation: $T_{a}+T_{b}+T_{c}=0$. Referring to the data in Tab.2, this equality holds at $t_{2}$ and $t_{4}$, but does not at $t_{1}$ and $t_{3}$. Comparing the $\left|T_{b} / T_{a}\right|$ values of these moments, the $t_{2}\left|T_{b} / T_{a}\right|$ and $t_{4}\left|T_{b} / T_{a}\right|$ equal to $K$, but $t_{1}\left|T_{b} / T_{a}\right|$ and $t_{3}\left|T_{b} / T_{a}\right|$ have no relation with $K$. It is sure that ratio of torques is not consistent with the ratio of static analysis result, unless the system entered into stable period.

Tab. 2 torques of 4 periods

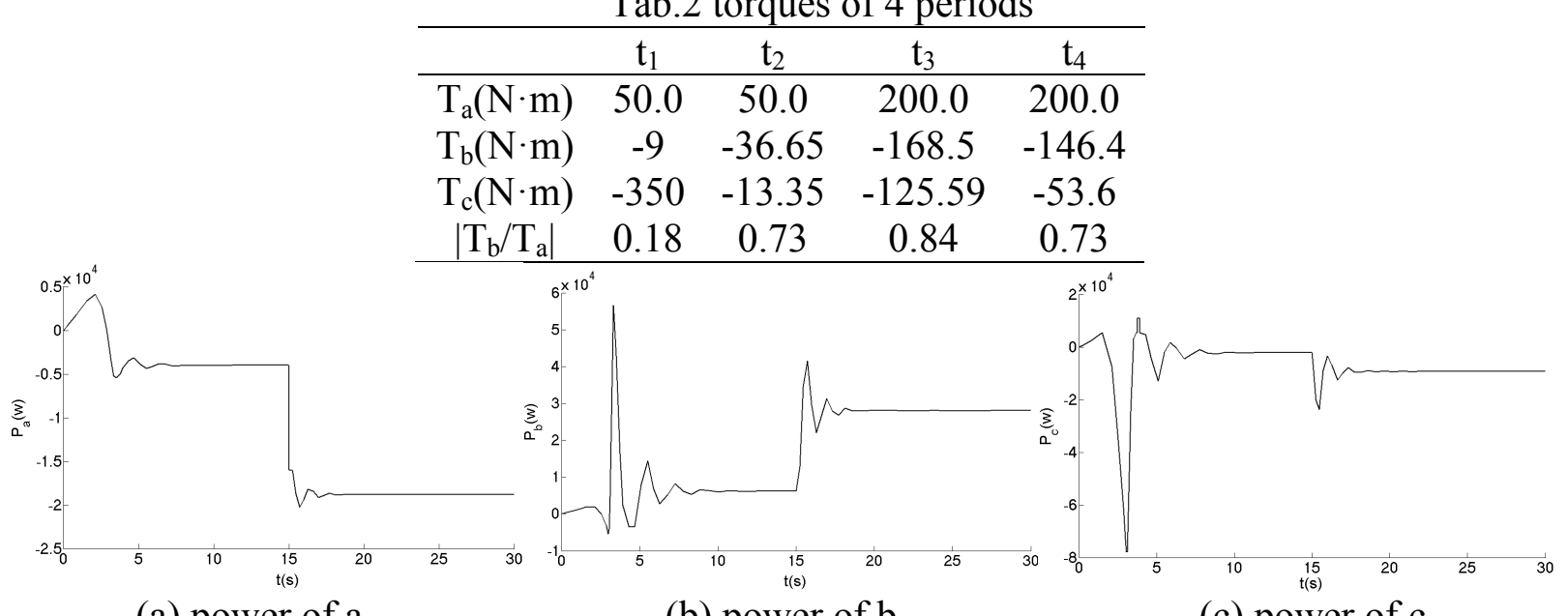

(a) power of a

(b) power of b

(c) power of c

Fig.4 power of PGT's components

Figure 4 shows the power curve of each component of the PGT. The power curve shows, components $\mathrm{a}$ and $\mathrm{c}$ are loads, and component $\mathrm{b}$ is motor. The angular velocity diagram shown in Figure 2, angular velocity in the opposite direction of components a and $\mathrm{c}$, a and b have the same angular velocity direction. Torque diagram as shown in Figure 3, torque in the opposite direction of components a and $\mathrm{c}, \mathrm{b}$ and $\mathrm{c}$ have the same torque direction. It cannot only be a simple analysis of the angular velocity or torque direction as the input or output port to PGT, but also the both of these two direction parameters. That is, the power of positive and negative as a basis for judging which component is input or output port. This method of judgment is also used to judge the power direction of power split and confluence.

Tab.3 power and power distribution of stable periods

\begin{tabular}{cccccc}
\hline & $\mathrm{P}_{\mathrm{a}}(\mathrm{w})$ & $\mathrm{P}_{\mathrm{b}}(\mathrm{w})$ & $\mathrm{P}_{\mathrm{c}}(\mathrm{w})$ & $\left|\mathrm{P}_{\mathrm{a}} / \mathrm{P}_{\mathrm{b}}\right|(\%)$ & $\left|\mathrm{P}_{\mathrm{c}} / \mathrm{P}_{\mathrm{b}}\right|(\%)$ \\
\hline First stable period & $-4.0 \times 10^{3}$ & $6.16 \times 10^{3}$ & $-2.16 \times 10^{3}$ & 64.94 & 35.06 \\
Second stable period & $-1.88 \times 10^{4}$ & $2.804 \times 10^{4}$ & $-9.25 \times 10^{3}$ & 67.04 & 32.98 \\
\hline
\end{tabular}

As the first and third periods of the system are in an unstable state, power becomes unstable too. Table 3 extracts the power of each component in the two stable periods to analyze power distribution after the stable period. Because the $b$ component corresponding to the $b$ port is power input port, $\left|\mathrm{P}_{\mathrm{a}} / \mathrm{P}_{\mathrm{b}}\right|,\left|\mathrm{P}_{\mathrm{c}} / \mathrm{P}_{\mathrm{b}}\right|$ expressed as power percentages which distributed by a and c port. From table 3, we know that, even within the same stabilization period, in conditions of speed and torque changing, power distribution has changed.

\section{Summary}

Based on the dynamic analysis method for analysis of the PGT dynamic response under two conditions which contain the boot and load status, the results can prove that the behavior of dynamic and static analysis are not exactly the same. In the dynamic process, the ratio of angular velocities is always consistent with the parameter $\mathrm{k}$; the ratio of torque is consistent with the parameter $\mathrm{k}$ only during the stabilizing period, but it will change greatly during the non-stabilizing period which is influenced by interference or load mutation.

So, in planetary gear train research, results of static analysis are not always completely accurate in all cases. When the research object is applied to changed frequently conditions or in precision mechanism, corresponding torque change should be considered. Power is associated with torque and rotational speed at the same time, so, if you want to get the right power distribution result in 
gear train during operation, speed and torque must be considered in real time status simultaneously.

This thesis gets the conclusions through dynamic analysis method for dynamic response analysis of the planetary gear train, these conclusions will provide reference for further compound gear train research.

\section{Acknowledgment}

The authors appreciate the fund of National Natural Science Foundation of People's Republic of China (No. 51175419 ). Corresponding author: Yahui Cui: E-mail address: cyhxut@xaut.edu.cn

\section{References}

[1] CUI Yahui. Study on the bifurcated power planetary transmission (in Chinese). Xi'an: Xi'an University of Technology, 1998.

[2] XU Hailiang. Theoretical and theory research on single-loop planetary transmission (in Chinese). Xi'an: Xi'an University of Technology, 2010.

[3] SUN Zhenzhong. Theoretical analysis and experimental study of differential gear train for parallel hybrid electric vehicle (in Chinese). Xi'an: Xi'an University of Technology, 2010.

[4] LUO Y T, TAN D, Dynamic modeling of planetary gear set considering meshing stiffness based on bond graph. Procedia Engineering, 2011, 24:850-855.

[5] QIN Datong, GU Xiguo, Wang Jianhong, et al. Dynamic analysis and optimization of gear trains in a megawatt level wind turbine (in Chinese).Journal of Chongqing University (Natural Science Edition), 2009, 32(4): 408-414

[6] LIN J, PARKER R G. Analytical characterization of the unique properties of planetary gear free vibration. Journal of Vibration and Acoustics, 1999, 121:316-321.

[7] LIN J, PARKER R G. Planetary gear parametric instability caused by mesh stiffness variation. Journal of Sound and Vibration, 2002, 249(1):129-145.

[8] BAHK C J, PARKER R G. Analytical solution for the nonlinear dynamics of planetary gears. Journal of Computationl and Nonlinear dynamics, 2011, 6:021007-1-15.

[9] BAHK C J, PARKER R G, A study on planetary gear dynamics with tooth profile modification // Proceedings of the ASME 2011 International Design Engineering Technical Conferences \& Computers and Information in Engineering Conference, 2011, Washington DC. New York: ASME, 2011:47346-1-13.

[10]SUN Zhimin, JI Linhong, SHEN Yunwen. Nonlinear dynamics of $2 \mathrm{~K}-\mathrm{H}$ planetary gear train (in Chinese). J. Tsinghua Univ., 2003, 43(5):636-639.

[11]LIU Zhenhao, WU Shijing, WANG Xiaosun, et al. Nonlinear dynamics of compound planetary gear sets based on incremental harmonic balance method (in Chinese). Journal of Vibration and Shock, 2012, 31(3):117-122.

[12]ZHU Enyong, WU Shijng, WANNG Xiaosun, et al. Study on nonlinear dynamic model of planetary gear train sets with friction force (in Chinese). Journal of Vibration and Shock, 2010, 29(8):217-220.

[13] GUO Y, PARKER R G. Dynamic analysis of planetary gears with bearing clearance. Journal of Computational and Nonlinear Dynamics, 2012, 7: 041002-1-15.

[14]GUO Y, KELLER J, PARKER R G. Dynamic analysis of planetary gears with bearing clearance. European Journal of Mechanics A/Solids, 2014, 47: 45-57. 
[15]L. MANGIALARDI, G. MANTRIOTA. Power flows and efficiency in infinitely variable transmissions. Mechanism and Machine Theory 1999, 34: 973-994.

[16]ZHANG Ce. Machinery dynamics (in Chinese). Beijing: Higher Education Press, 2008. 